\title{
The Retention of Proprioceptive Information is Suppressed by Competing Verbal and Spatial, but not by Motor and Visual Tasks
}

Áron Horváth ( $\square$ horvath.aron@ppk.elte.hu )

ELTE Eötvös Loránd University

Eszter Ferentzi

ELTE Eötvös Loránd University

Anett Ragó

ELTE Eötvös Loránd University

Ferenc Köteles

ELTE Eötvös Loránd University

\section{Research Article}

Keywords: proprioception, proprioceptive accuracy, proprioceptive memory, working memory, short-term memory, interference

Posted Date: July 2nd, 2021

DOI: https://doi.org/10.21203/rs.3.rs-644604/v1

License: (c) (i) This work is licensed under a Creative Commons Attribution 4.0 International License. Read Full License 


\section{Abstract}

Proprioceptive information, originating from the locomotor system makes us able to perceive the position of our joints from an internal point of view. In certain cases, proprioceptive information has to be stored in short-term memory, for example, during the learning of new motor skills or the assessment of proprioceptive accuracy. However, there are contradictory findings about the modality-specific storage of proprioceptive information in working memory. In this preregistered study, we applied the interference paradigm, assessing proprioceptive memory capacity in the subdominant elbow joint for 35 young individuals in five different experimental conditions: (a) without competing task/suppression (control condition), (b) with motor suppression, (c) with spatial suppression, (d) with visual suppression, and (e) with verbal suppression. Proprioceptive span was lower in the verbal and spatial suppression condition than in the control condition, whereas no differences were found for the remaining conditions. These results indicate that individuals use verbal and spatial strategies to encode proprioceptive information in short-term memory, and, in contrast to our expectation, the motor subsystem of working memory is not involved in this process.

\section{Introduction}

Certain types of mechanoreceptors, located in our locomotor system (i.e muscles, joints, and ligaments) make us able to perceive the position and movement of our body, and to sense force and heaviness ${ }^{1}$. This ability, called proprioception, plays a prominent role in movement regulation, along with other sensory modalities, such as vision and tactile sensation ${ }^{2,3}$. Motor control typically relies on online proprioceptive feedback ${ }^{2}$. Sometimes, however, the idea that this information is stored in short-term memory ${ }^{4}$. For example, the learning of new motor sequences in sports or everyday activities may require the ability to store and recall proprioceptive information for short term. Despite its practical and theoretical importance, the exact mechanism of this process, i.e., how proprioceptive information is stored in short-term memory, has gained little attention to date.

While teaching new motor skills, instructors often show the correct movement by grabbing athletes' body parts, and moving them in the desired pattern ${ }^{5}$. This way of teaching proved to be effective: learning a movement trajectory by presenting it with passively moving the arm is more effective than learning by relying purely on visual presentation ${ }^{6}$. In order to effectively execute the desired movement, one has to accurately perceive the proprioceptively presented joint positions, store them in short term memory, and reproduce the entire sequence by active motion. When movement sequences are complex (containing several joint positions), one's ability to store proprioceptive information in memory may limit the quality of movement reproduction and eventually motor learning.

The storage of proprioceptive information is necessary for most of the tests that measure proprioceptive accuracy, i.e., the acuity of perception of the position of the joints. For example, in the ipsilateral version of the Joint Position Reproduction test (JPR), the limb of participants is set to a target position, then moved away from it, and participants are asked to replicate the target position as accurately as possible. 
To do so, the target position needs to be stored in short term memory ${ }^{4}$. Cognitive factors, such as attentional load 7,8 and working memory capacity ${ }^{9}$ were proved to influence the outcome of the task, which supports the idea that short term memory is involved in the process. Change in accuracy may also help to evaluate the feasibility an intervention ${ }^{10}$, and can be used for sport selection ${ }^{11}$ and injury prevention ${ }^{12}$. To be able to draw valid and reliable conclusions, it is important to explore the factors that could influence the outcome of the JPR test.

Based on interference studies, one can consider the four modality-specific subsystems belonging to working memory as possible modulation factors. These subsystems store and reproduce verbal (e.g., word lists), spatial (e.g., sequences of spatial positions), visual (e.g., complex figures) or motor (e.g., body-related movements) stimuli ${ }^{13-15}$. The involvement of these subsystems in a certain task is typically studied with the so-called interference paradigm. The general assumption is that if the rivalry of the two modalities does not disrupt theirs retention, then the dual task activates two separate and independent subsystems of the working memory ${ }^{16}$. For example, spatial suppression (e.g. repeatedly pointing to spatial positions) substantially disrupts the spatial memory performance but does no influence the verbal, motor or visual memory performance ${ }^{15-17}$. The existence of the independent subsystems is further supported by correlation studies where capacity measures of different modalities were shown to not overlap ${ }^{18,19}$.

The question that which modality-specific subsystem of the short term memory stores proprioceptive information is investigated rarely, and studies have equivocal or even contradictory results. Goble and colleagues ${ }^{20}$ found that cerebral palsy patients could improve their accuracy in the JPR task if joint position were presented for a longer time ( $15 \mathrm{sec})$, compared to short time presentation $(2 \mathrm{sec})$. As the magnitude of this improvement showed a positive relationship with the spatial memory span (assessed with the Corsi task) of the patients, the authors concluded that spatial memory plays an inherent role in storing joint position-related proprioceptive information. However, this conclusion was not supported by the findings of another study. Horváth and colleagues ${ }^{18}$ investigated the association between proprioceptive span (i.e. the maximal number of proprioceptively determined joint positions that one can store in short-term memory) and verbal or spatial short-term memory span (assessed with the digit span task and the Corsi task, respectively) in a sample of university students. Proprioceptive span proved to be independent of verbal and spatial spans, which suggests that proprioceptive information might be stored in another subsystem.

The primary goal of the present study was to explore the modality-specific storage of proprioceptive information in short-term memory. Our hypothesis was that people store a series of proprioceptively determined joint position in the same way as visually observed movement sequences, i.e., in a motor form ${ }^{15,21}$. Thus, executing a motor suppression task while encoding sequences of joint positions should be associated with decreased performance, whereas other tasks (verbal, spatial, visual) would not impact it. For this purpose, we adapted and modified the task used by Horváth and colleagues ${ }^{18}$ to assess proprioceptive short-term memory span in a within-subject research design with five different 
experimental conditions: (1) without competing task/suppression (control condition), (2) with motor suppression, (3) with verbal suppression, (4) with spatial suppression, and (5) with visual suppression.

\section{Methods}

Our sample size, hypothesis, study design and analyses was preregistered (available at https://osf.io/qx9me). The raw data and statistical analysis are also publicly available (https://osf.io/yvu97/).

\section{Participants}

We used the G*Power (version: 3.1 .9 .4 ) software to a priori determine sample size. Based on previous similar (suppression) studies ${ }^{15,22}$, effect size was set to large (partial eta square $=0.14$ ). To achieve an alpha of 0.05 and a power of 0.95 , the required minimum sample size is 31 for a repeated measures ANOVA with 5 levels. Based on this, our a priori decision was to stop when $\mathrm{N}=35$ is reached. Overall, 35 undergraduate students of the Eötvös Loránd University completed the measurements ( 25 women, 33 right-handed). Participants were at least 18 years old (mean age was $21.2 \pm 3.05$ ), without severe injury or disability of the elbow joint. In average, participants spend $2.9 \pm 2.5$ hours/week with sporting activity (e.g. running, calisthenics). They received partial course credit for the participation. The experiment was approved by the Research Ethics Committee of the Faculty of Education and Psychology of the Eötvös Loránd University (approval number: 2019/302-2). Every participant signed the informed consent before the experiment. All tasks were performed in accordance with the relevant guidelines and regulations.

\section{Capacity measurement}

To measure proprioceptive memory span, we adapted and modified the task developed by Horváth and colleagues ${ }^{18}$ for assessing the ability to memorize and reproduce sequences of elbow joint positions. For the assessment, a custom-made motor-driven device (proprioceptor) was used, which enabled us to accurately set $( \pm 0.5$ degree $)$ and measure $( \pm 0.1$ degree $)$ the angle of the elbow joint. Quasi-random sequences of different lengths were composed from nine possible target positions $(30,45,60,75,90,105$, $120,135,150$ degrees, where the higher values refer to the bigger extension of the elbow joint). Every target position was presented only once in a sequence (until the length reached 10 positions). The starting position of the trials was always the same, i.e. an almost fully extended elbow (160 degree). From there, the device started to move the elbow joint of the participant, then stopped the movement and kept the arm for 4 seconds in every target position. Target positions were presented directly after each other without returning to the starting position. After the presentation of an entire sequence, the proprioceptor moved back the elbow joint to the starting position; form this position, participants were asked to replicate the whole sequence by actively moving their arm and pressing a button at every target position. The measurement started with three 2-position practice sequences; than the assessment started 
with 3-position sequences. If one correctly reproduced two sequences of a given length out of a maximum of three attempts, the number of presented positions increased by one in the next sequence. However, if sequences of the given length were reproduced incorrectly twice, the task ended. The capacity score was determined by the number of elements in the longest, at least two times correctly reproduced sequence. The given sequence was considered correct if: (1) the movement pattern was correct (no more or fewer positions were reproduced, and no movement were performed to the opposite direction), and (2) the difference between the target and the reproduced position was less than $30^{\circ}$ in each case.

\section{Procedure}

Every participant performed the proprioceptive memory capacity measurement in five different conditions: (a) no suppression (control), (b) motor suppression, (c) spatial suppression, (d) visual suppression, and (e) verbal suppression (see Figure 1). The competing tasks were administered during the presentation phase of the proprioceptive measurement only. The no suppression (control) condition was administered first, followed by the remaining four conditions in a randomized order.

\section{Control}

During the control measurement, participants had no competing task, so they could fully concentrate on the proprioceptive memory task. Thus, this task measured the memory span of participants.

\section{Motor suppression}

Motor suppression task was adapted from Smyth and colleagues ${ }^{15}$. Participants had to repeatedly touch their body parts with their dominant hand in the following order: left shoulder, right shoulder, left hip, right hip. This was presented by the experimenter at a speed of approximately 4 touch/second, and participants were instructed to keep that speed.

\section{Spatial suppression}

This task was adapted from Smyth and colleagues ${ }^{15}$. Participants had to repeatedly touch spatial positions with eyes closed, represented by rectangular boxes (width: $3.5 \mathrm{~cm}$, length: $5 \mathrm{~cm}$, height: $1.5 \mathrm{~cm}$ ), aligned in a square layout, with $2.5 \mathrm{~cm}$ space between them. Participants had to touch the top of the boxes. This was presented by the experimenter with approximately a 4 box/second speed, and participants were asked to try to keep that rhythm.

\section{Visual suppression}


The visual suppression task was adapted from Della Sala and colleagues ${ }^{17}$. Participants had to watch abstract pictures on a laptop screen (e.g. pictures of Wassily Kandinsky or Jackson Pollock). The sight of the tested arm was blocked by a specific eye-mask. Each picture was presented for 5 seconds.

\section{Verbal suppression}

The verbal suppression task was adapted from Baddeley and colleagues ${ }^{23}$. Participants had to repeatedly count from one to four aloud. The task was presented by the experimenter with approximately a four digit per second speed, and they were asked to keep that speed.

\section{Statistical analysis}

Statistical analysis was conducted in the JAMOVI (version: 1.6) software ${ }^{24}$. The assumptions of repeated ANOVA were not met, as the Shapiro-Wilk test of normality was significant in every condition $(p<0.05)$. To compare the experimental conditions, we used repeated measures Friedman test with 5 levels. Durbin-Conover test was used for the post hoc analysis.

\section{Results}

Descriptive statistics of the investigated variables are presented in Table 1.

Table 1

Descriptive statistics of participants' proprioceptive span in the five conditions

\begin{tabular}{|llllll|}
\hline Condition & Median & Mean & Standard deviation & Minimum & Maximum \\
\hline control & 6 & 5.69 & 1.08 & 3 & 8 \\
\hline motor & 5 & 5.26 & 1.70 & 3 & 10 \\
\hline spatial & 5 & 4.97 & 1.36 & 3 & 9 \\
\hline visual & 5 & 5.31 & 1.35 & 3 & 8 \\
\hline verbal & 4 & 4.83 & 1.65 & 3 & 9 \\
\hline
\end{tabular}

\section{Hypothesis testing}

The repeated measures Friedman test indicated a significant difference between the conditions $\left(\chi^{2}=13.3\right.$, $p=0.01$ ). The Durbin-Conover test showed that proprioceptive span was significantly lower in the verbal 
condition than in the control condition $(p<0.001)$, and also significantly lower in the spatial condition than in the control condition ( $p=0.006)$ (Fig. 2).

\section{Discussion}

In this study, we investigated the modality-specific storage of proprioceptive information in short-term memory by measuring participants' ability to reproduce sequences of proprioceptively determined joint positions (i.e., proprioceptive span) while executing competing verbal, visual, spatial, or motor tasks. We hypothesized that proprioceptive information is stored in a motor form, thus we predicted that motor suppression would decrease proprioceptive span, while verbal, visual, and spatial suppression would not. In contrast to our expectation, our findings show that competing verbal and spatial tasks had a negative impact on proprioceptive span, whereas visual and motor suppression effect did not occur. Overall, theses results suggest that people typically use verbal and/or spatial strategies when they need to store a series of proprioceptively determined spatial position.

The results of Goble and colleagues, $2012^{20}$, that showed a positive association between spatial memory span and improvement in proprioceptive accuracy for longer presentation time of joint positions in patients with cerebral palsy, is partly in accordance with this conclusion. In contrast, proprioceptive memory capacity did not correlate either with spatial or verbal memory capacity in the study of Horváth and colleagues, $2020^{18}$. These differences can be explained by multiple reasons. First, there are methodological differences between the studies: Goble and colleagues, $2012{ }^{20}$ used a correlational design, but the involvement of verbal, motor, and visual short-term memory was not tested. Horváth and colleagues, $2020^{18}$ also conducted a correlational study, however, they did not test the involvement of visual and motor short-term memory. The present study applied an experimental (interference) design, and all possible modality-specific subsystems (i.e., motor, spatial, visual, and verbal) were tested. Second, it is important to note that there is no association between proprioceptive span (the maximal number of joint positions one can retain is short-term memory) and proprioceptive accuracy (the ability to store one joint position as accurately as possible) ${ }^{18}$. It is possible that different mechanisms are responsible for storing a single joint position (as in Goble and colleagues, $2012^{20}$ ) or to store a maximal number of joint positions (as in the Horváth and colleagues, $2020^{18}$ and the present study). A third possible cause behind the equivocal results may be the difference between the investigated samples. It was shown that processing of stimuli in working-memory can depend on motor expertise ${ }^{22}$. While elite athletes encode visually observed movement sequences in a motor form, non-athletes use verbal form ${ }^{22}$. Also, elite athletes utilize motor strategies for mental rotation, while non-athletes do not ${ }^{25}$. Based on these findings, the involvement of motor short-term memory in storing proprioceptively determined joint position sequences may also depend on the motor expertise of the participants. Thus, the results of the current study may be specific to people spending relatively little time with sporting activity $(2.9 \pm 2.47$ hours/week). The contradictory findings of Horváth and colleagues, $2020^{18}$, namely the independence of proprioceptive, verbal, and spatial spans, may be explained by participants' significantly higher physical 
activity (8.0 \pm 3.4 hours/week), which indicates a higher level of motor expertise. Table 2 . summarizes the most pivotal differences between these studies.

Table 2

Summary of the differences between the articles investigating the modality-specific storage of proprioceptive information

\begin{tabular}{|c|c|c|c|}
\hline Study & $\begin{array}{l}\text { Goble et al. } \\
2012^{20}\end{array}$ & Horváth et al. $2020^{18}$ & Present study \\
\hline Assessed variable & $\begin{array}{l}\text { Proprioceptive } \\
\text { accuracy }\end{array}$ & Proprioceptive span & Proprioceptive span \\
\hline Sample & $\begin{array}{l}\text { Cerebral palsy } \\
\text { patients }\end{array}$ & $\begin{array}{l}\text { University students } \\
\text { (sporting } 8.0 \pm 4.0 \\
\text { hours/week) }\end{array}$ & $\begin{array}{l}\text { University students (sporting } \\
2.9 \pm 2.5 \text { hours/week) }\end{array}$ \\
\hline Study design & correlational & correlational & interference-based \\
\hline $\begin{array}{l}\text { Subsystem found } \\
\text { to be involved }\end{array}$ & spatial & - & spatial, verbal \\
\hline $\begin{array}{l}\text { Subsystem found } \\
\text { not to be involved }\end{array}$ & - & verbal, spatial & motor, visual \\
\hline $\begin{array}{l}\text { Subsystem not } \\
\text { investigated }\end{array}$ & $\begin{array}{l}\text { verbal, motor, } \\
\text { visual }\end{array}$ & motor, visual & - \\
\hline
\end{tabular}

From a theoretical point of view, our findings also do no support the idea that people use the motor subsystem in short-term memory to store proprioceptive stimuli. This can be explained by the two-step process of motor learning. Conscious awareness and voluntary motor control are involved in the first stage only ${ }^{26,27}$. In this initial phase, the use of the verbal modality (i.e., in the form of secondary representation, perhaps also supported by the spatial module of short-term memory) appears sufficient. In the second phase, patterns of movements are stored in procedural memory and executed automatically 26,27 , which does not require a modality-specific subsystem of short-term memory.

Our findings may have important practical consequences related to the field of athletic training too. When teaching new motor sequences with proprioceptive presentation, it is important to consider that there are individual differences in the capacity to store joint positions in working memory. Thus, the ideal number of the presented joint positions depends on the individual. As the process utilizes the verbal and spatial systems, it could be helpful to find the appropriate verbal labels and spatial strategies.

Our study is not without limitations. We assessed proprioceptive memory capacity only in the nondominant hand; it cannot be excluded that proprioceptive sequences are stored differently in the case of the dominant hand. Also, as mentioned before, the results might be specific to the studied population (university students with comparatively low level of physical activity). Further, we used a relatively liberal decision criterion $\left(<30^{\circ}\right)$ with respect to the acceptable difference between the target and the reproduced position in the measurement of proprioceptive memory span. 
The strength of this study is that all considerable short-term memory subsystems (motor, spatial, visual, verbal) were investigated with a unitary experimental (interference) design. Further studies may be required to explore the possible differences between athletes and non-athletes in the retention of proprioceptive information. It would be also valuable to test how the capacity to store proprioceptive information can be improved, and how it affects motor learning.

\section{Declarations}

\section{Acknowledgements}

Supported by the ÚNKP-20-3 New National Excellence Program of the Ministry for Innovation and Technology from the source of the National Research, Development and Innovation Fund (ÚNKP-20-3-IIELTE-163).

\section{Conflicts of Interest and Source of Funding}

Supported by the ÚNKP-20-3 New National Excellence Program of the Ministry for Innovation and Technology from the source of the National Research, Development and Innovation Fund (ÚNKP-203-II-ELTE-163).

All authors declare that there is no conflict of interest.

\section{Author contributions}

All authors took part in the conceptualization of the study. ÁH wrote the first draft of the manuscript. EF, AR and FK wrote sections of the manuscript. FK supervised the project. All authors reviewed the manuscript.

\section{References}

1. Proske, U. \& Gandevia, S. C. The proprioceptive senses: their roles in signaling body shape, body position and movement, and muscle force. Physiol. Rev, 92, 1651-1697 (2012).

2. Goodman, R. \& Tremblay, L. Using proprioception to control ongoing actions: dominance of vision or altered proprioceptive weighing? Exp Brain Res, 236, 1897-1910 (2018).

3. Veilleux, L. N. \& Proteau, L. Congruent visual and proprioceptive information results in a better encoding of initial hand position. Exp Brain Res, 214, 215-224 (2011).

4. Goble, D. J. Proprioceptive acuity assessment via joint position matching: From basic science to general practice. Phys. Ther, 90, 1176-1184 (2010).

5. Chiyohara, S., Furukawa, J., Noda, T., Morimoto, J. \& Imamizu, H. Passive training with upper extremity exoskeleton robot affects proprioceptive acuity and performance of motor learning. Sci. 
Rep, 10, 11820 (2020).

6. Wong, J. D., Kistemaker, D. A., Chin, A. \& Gribble, P. L. Can proprioceptive training improve motor learning? Journal of Neurophysiology, 108, 3313-3321 (2012).

7. Boisgontier, M. P., Olivier, I., Chenu, O. \& Nougier, V. Presbypropria: the effects of physiological ageing on proprioceptive control. Age (Dordr), 34, 1179-1194 (2012).

8. Yasuda, K., Sato, Y., limura, N. \& Iwata, H. Allocation of Attentional Resources toward a Secondary Cognitive Task Leads to Compromised Ankle Proprioceptive Performance in Healthy Young Adults. Rehabilitation Research and Practice, https://doi.org/10.1155/2014/170304 (2014).

9. Goble, D. J., Mousigian, M. A. \& Brown, S. H. Compromised encoding of proprioceptively determined joint angles in older adults: the role of working memory and attentional load. Exp Brain Res, 216, 3540 (2011).

10. Isaac, S. M. et al. Does arthroplasty type influence knee joint proprioception? A longitudinal prospective study comparing total and unicompartmental arthroplasty. Knee, 14, 212-217 (2007).

11. Han, J., Waddington, G., Anson, J. \& Adams, R. Level of competitive success achieved by elite athletes and multi-joint proprioceptive ability. Journal of Science \& Medicine in Sport, 18, 77-81 (2015).

12. Cameron, M., Adams, R. \& Maher, C. Motor control and strength as predictors of hamstring injury in elite players of Australian football. Physical Therapy in Sport, 4, 159-166 (2003).

13. Baddeley, A. D. \& Logie, R. H. Working memory: The multiple-component model. in Models of working memory: Mechanisms of active maintenance and executive control 28-61(Cambridge University Press, 1999). doi:10.1017/СВ09781139174909.005.

14. Klauer, K. C. \& Zhao, Z. Double Dissociations in Visual and Spatial Short-Term Memory. Journal of Experimental Psychology: General, 133, 355-381 (2004).

15. Smyth, M. M., Pearson, N. A. \& Pendleton, L. R. Movement and Working Memory: Patterns and Positions in Space. The Quarterly Journal of Experimental Psychology Section A, 40, 497-514 (1988).

16. Baddeley, A. D. Working memory., 255, 556-559 (1992).

17. Della Sala, S., Gray, C., Baddeley, A., Allamano, N. \& Wilson, L. Pattern span: a tool for unwelding visuo-spatial memory., 37, 1189-1199 (1999).

18. Horváth, Ã., Ragó, A., Ferentzi, E., Körmendi, J. \& Köteles, F. Short-term retention of proprioceptive information. Quarterly Journal of Experimental Psychology, 73, 2148-2157 (2020).

19. Ichikawa, S. Verbal memory span, visual memory span, and their correlations with cognitive tasks. Jpn. Psychol. Res, 25, 173-180 (1983).

20. Goble, D. J., Aaron, M. B., Warschausky, S., Kaufman, J. N. \& Hurvitz, E. A. The influence of spatial working memory on ipsilateral remembered proprioceptive matching in adults with cerebral palsy. Exp Brain Res, 223, 259-269 (2012). 
21. Smyth, M. M. \& Pendleton, L. R. Space and Movement in Working Memory. The Quarterly Journal of Experimental Psychology Section A, 42, 291-304 (1990).

22. Moreau, D. Motor expertise modulates movement processing in working memory. Acta. Psychol, 142 , 356-361 (2013).

23. Baddeley, A. D., Thomson, N. \& Buchanan, M. Word length and the structure of short-term memory. Journal of Verbal Learning and Verbal Behavior, 14, 575-589 (1975).

24. The jamovi project. jamovi. (Version 1.6) [Computer software] Retrieved from https://www.jamovi.org. (2021).

25. Moreau, D. The role of motor processes in three-dimensional mental rotation: Shaping cognitive processing via sensorimotor experience. Learning and Individual Differences, 22, 354-359 (2012).

26. Gentile, A. M. Movement Science: Implicit and Explicit Processes during Acquisition of Functional Skills. Scandinavian Journal of Occupational Therapy, 5, 7-16 (1998).

27. Lusardi, M. M. \& Bowers, D. M. Motor Control, Motor Learning, and Neural Plasticity in Orthotic and Prosthetic Rehabilitation(2013).

\section{Figures}




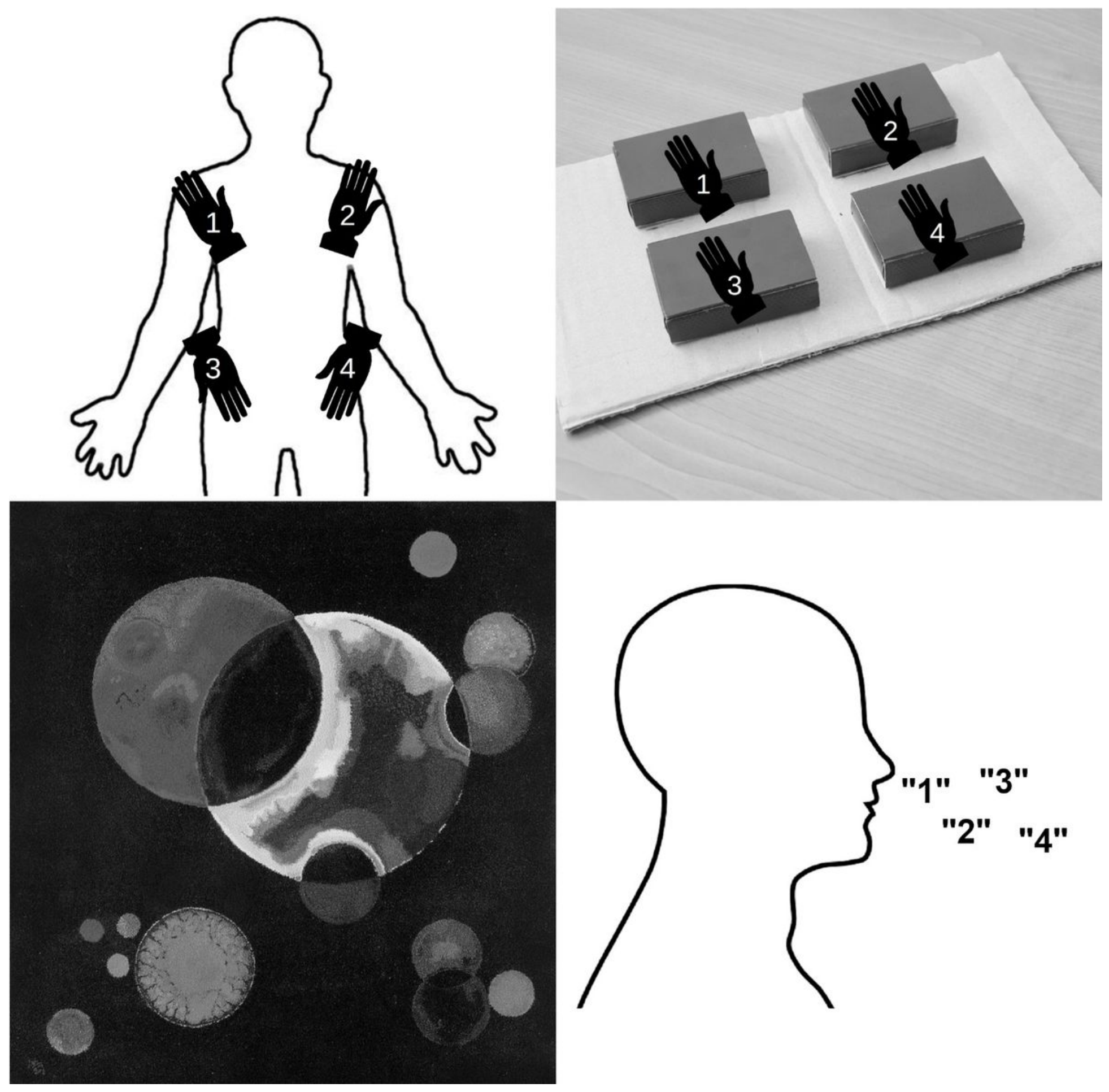

Figure 1

Illustration of the four competing tasks. Motor suppression: repeated touching of four body parts (upper left); Spatial suppression: repeated touching of four objects with closed eyes (upper right); Visual suppression: looking at abstract images (lower left); Verbal suppression: repeated counting from one to four (lower right) 


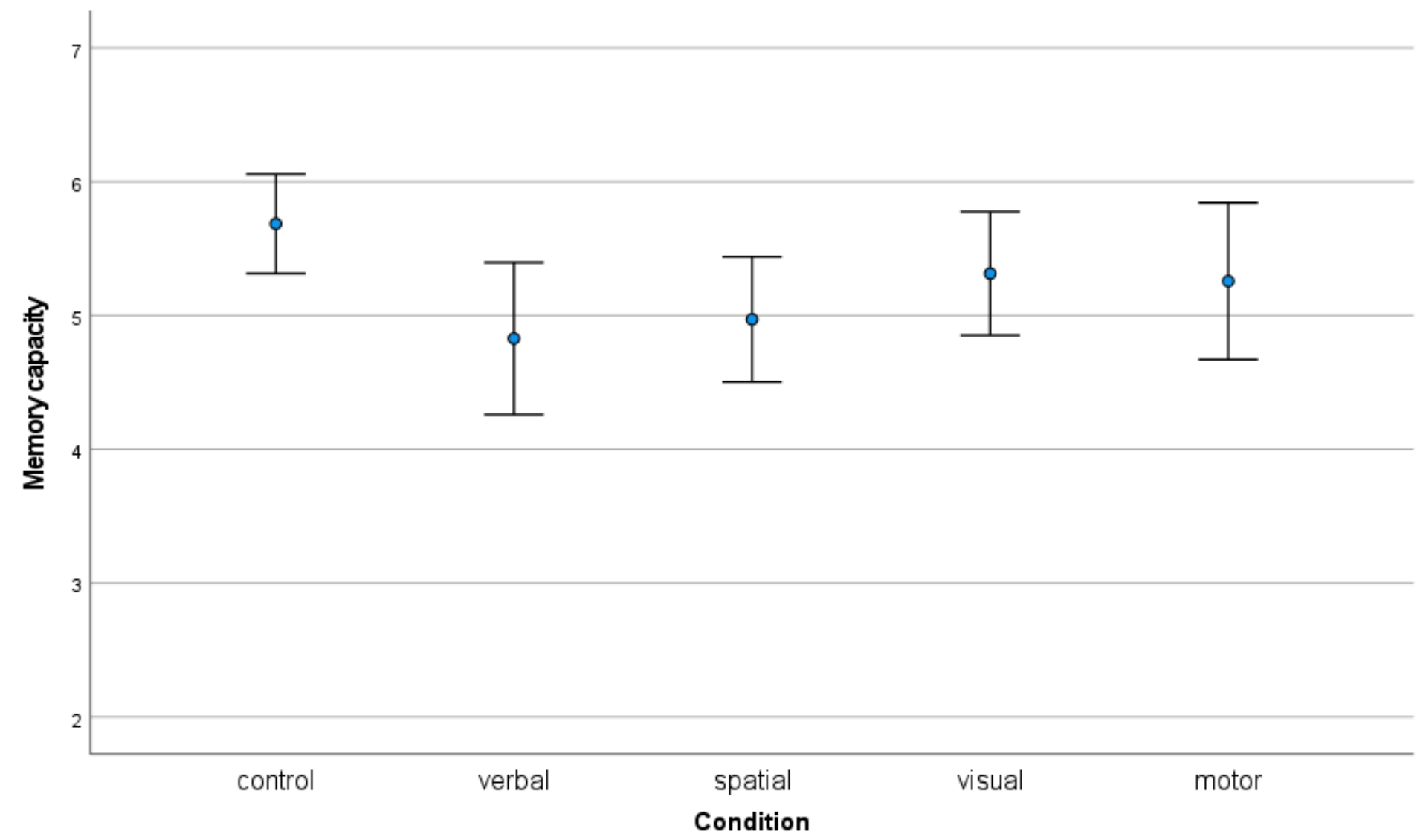

Figure 2

Mean capacity scores in the different experimental conditions. Error bars represent $95 \%$ confidence intervals. 